\title{
Correction to: Avoiding taxes: banks' use of internal debt
}

\author{
Franz Reiter ${ }^{1} \cdot$ Dominika Langenmayr $^{2} \oplus \cdot$ Svea Holtmann ${ }^{3}$
}

Published online: 9 January 2021

(c) Springer Science+Business Media, LLC, part of Springer Nature 2021

\section{Correction to: International Tax and Public Finance https://doi.org/10.1007/s10797-020-09625-2}

The original version of this article contained a mistake in the table 4 . The table 4 labelling is incorrect. The correct Table 4 labelling for "Dep. var." should read as follow: 1st column as IntLiab/TA and 2nd, 3rd, 4th column as IntNetDebt/TA, 5th column IntLiab/TA and 6th, 7th and 8th column as IntNetDebt/TA. The correct Table 4 is given below.

The original article can be found online at https://doi.org/10.1007/s10797-020-09625-2.

Dominika Langenmayr

dominika.langenmayr@ku.de

Franz Reiter

franz.reiter@mail.de

Svea Holtmann

sholtmann@ku.de

1 University of Munich, Akademiestr. 1/II, 80799 Munich, Germany

2 KU Eichstätt-Ingolstadt and CESifo, Auf der Schanz 49, 85049 Ingolstadt, Germany

3 KU Eichstätt-Ingolstadt, Auf der Schanz 49, 85049 Ingolstadt, Germany 
Table 4 Baseline intragroup debt regressions

\begin{tabular}{|c|c|c|c|c|c|c|c|c|}
\hline \multirow{3}{*}{$\begin{array}{l}\text { Sample } \\
\text { Dep. var. }\end{array}$} & \multicolumn{4}{|l|}{ All entities } & \multicolumn{4}{|c|}{ Foreign affiliates } \\
\hline & \multirow{2}{*}{$\begin{array}{l}\frac{\text { IntLiab }}{T A} \\
(1)\end{array}$} & \multicolumn{3}{|l|}{$\frac{\text { IntNetDebt }}{T A}$} & \multirow{2}{*}{$\begin{array}{l}\frac{\text { IntLiab }}{T A} \\
(5)\end{array}$} & \multicolumn{3}{|l|}{$\frac{\text { IntNetDebt}}{T A}$} \\
\hline & & $\overline{(2)}$ & (3) & (4) & & (6) & (7) & (8) \\
\hline CTR & $\begin{array}{l}0.508^{*} \\
(0.270)\end{array}$ & $\begin{array}{l}0.565^{* *} \\
(0.225)\end{array}$ & $\begin{array}{l}0.363 * * \\
(0.146)\end{array}$ & & $\begin{array}{l}0.468 * \\
(0.265)\end{array}$ & $\begin{array}{l}0.511^{* *} \\
(0.214)\end{array}$ & $\begin{array}{l}0.344 * * \\
(0.151)\end{array}$ & \\
\hline groupCTR & & & & $\begin{array}{l}-0.540^{* *} \\
(0.219)\end{array}$ & & & & $\begin{array}{l}-1.036^{* * *} \\
(0.252)\end{array}$ \\
\hline $\operatorname{Ln}(\mathrm{TA})$ & $\begin{array}{l}-0.024 * * * \\
(0.007)\end{array}$ & $\begin{array}{l}-0.015^{* * *} \\
(0.006)\end{array}$ & $\begin{array}{l}0.009 * * \\
(0.003)\end{array}$ & $\begin{array}{l}0.006^{*} \\
(0.003)\end{array}$ & $\begin{array}{l}-0.004 \\
(0.007)\end{array}$ & $\begin{array}{l}0.006 \\
(0.006)\end{array}$ & $\begin{array}{l}0.016^{* * *} \\
(0.004)\end{array}$ & $\begin{array}{l}0.013 * * * \\
(0.004)\end{array}$ \\
\hline $\operatorname{Ln}(\mathrm{GDP})$ & $\begin{array}{l}0.019 \\
(0.015)\end{array}$ & $\begin{array}{l}-0.000 \\
(0.012)\end{array}$ & $\begin{array}{l}0.010 \\
(0.023)\end{array}$ & $\begin{array}{l}-0.006 \\
(0.023)\end{array}$ & $\begin{array}{l}0.022 \\
(0.014)\end{array}$ & $\begin{array}{l}0.000 \\
(0.011)\end{array}$ & $\begin{array}{l}0.020 \\
(0.024)\end{array}$ & $\begin{array}{l}0.007 \\
(0.024)\end{array}$ \\
\hline Inflation & $\begin{array}{l}-0.007^{*} \\
(0.004)\end{array}$ & $\begin{array}{l}-0.005^{* *} \\
(0.003)\end{array}$ & $\begin{array}{l}-0.000 \\
(0.001)\end{array}$ & $\begin{array}{l}-0.000 \\
(0.001)\end{array}$ & $\begin{array}{l}-0.013^{* * *} \\
(0.004)\end{array}$ & $\begin{array}{l}-0.008^{* * *} \\
(0.003)\end{array}$ & $\begin{array}{l}0.000 \\
(0.000)\end{array}$ & $\begin{array}{l}-0.000 \\
(0.001)\end{array}$ \\
\hline $\begin{array}{l}\text { GDP } \\
\text { growth }\end{array}$ & $\begin{array}{l}-0.010^{* *} \\
(0.004)\end{array}$ & $\begin{array}{l}-0.008 * * \\
(0.004)\end{array}$ & $\begin{array}{l}-0.001 \\
(0.001)\end{array}$ & $\begin{array}{l}-0.001^{*} \\
(0.001)\end{array}$ & $\begin{array}{l}-0.011^{\text {**** }} \\
(0.004)\end{array}$ & $\begin{array}{l}-0.009 * * * \\
(0.003)\end{array}$ & $\begin{array}{l}-0.001 \\
(0.001)\end{array}$ & $\begin{array}{l}-0.001 \\
(0.001)\end{array}$ \\
\hline $\begin{array}{l}\text { Fin. sector } \\
\text { share }\end{array}$ & $\begin{array}{l}0.879 * * * \\
(0.318)\end{array}$ & $\begin{array}{l}0.102 \\
(0.230)\end{array}$ & $\begin{array}{l}1.409 * * * \\
(0.225)\end{array}$ & $\begin{array}{l}1.552 * * * \\
(0.239)\end{array}$ & $\begin{array}{l}0.495 \\
(0.310)\end{array}$ & $\begin{array}{l}-0.285 \\
(0.219)\end{array}$ & $\begin{array}{l}1.543 * * * \\
(0.227)\end{array}$ & $\begin{array}{l}1.744 * * * \\
(0.242)\end{array}$ \\
\hline Reg. index & $\begin{array}{l}-0.018^{* *} \\
(0.009)\end{array}$ & $\begin{array}{l}-0.005 \\
(0.008)\end{array}$ & & & $\begin{array}{l}0.003 \\
(0.008)\end{array}$ & $\begin{array}{l}0.018^{* * *} \\
(0.007)\end{array}$ & & \\
\hline Cap. req. & $\begin{array}{l}0.221 \\
(2.735)\end{array}$ & $\begin{array}{l}1.256 \\
(1.975)\end{array}$ & & & $\begin{array}{l}-0.544 \\
(2.451)\end{array}$ & $\begin{array}{l}-0.544 \\
(1.775)\end{array}$ & & \\
\hline Time FE & $\checkmark$ & $\checkmark$ & $\checkmark$ & $\checkmark$ & $\checkmark$ & $\checkmark$ & $\checkmark$ & $\checkmark$ \\
\hline $\begin{array}{l}\text { Bank } \\
\text { group FE }\end{array}$ & $\checkmark$ & $\checkmark$ & $\checkmark$ & $\checkmark$ & $\checkmark$ & $\checkmark$ & $\checkmark$ & $\checkmark$ \\
\hline Bank FE & & & $\checkmark$ & $\checkmark$ & & & $\checkmark$ & $\checkmark$ \\
\hline$R^{2}$ & 0.359 & 0.332 & 0.799 & 0.795 & 0.403 & 0.484 & 0.781 & 0.777 \\
\hline $\begin{array}{c}\text { Observa- } \\
\text { tions }\end{array}$ & 21,896 & 21,896 & 21,893 & 21,884 & 16,187 & 16,187 & 16,184 & 16,181 \\
\hline
\end{tabular}

Dependent variable is the ratio of internal liabilities to total external assets in columns (1) and (4) and the ratio of internal net debt (internal liabilities net of internal claims if positive, zero otherwise) to total external assets in the other columns. groupCTR is the average tax rate of the bank group. Fin. sector share is the share of the banking and insurance sector in a country's gross value added. Reg. index captures the stringency of capital regulation in a country, ranging from 0 to 10 (higher values indicating greater stringency). Cap. req. is the legal minimum capital requirement for banks in a country. Standard errors in parentheses, clustered by bank and by country-month. ***,*** indicate significance at the $1 \%$, 5\%, 10\% levels. Regressions based on monthly data for 06/2010-12/2015 from the External Positions of Banks database of Deutsche Bundesbank (2015)

Publisher's Note Springer Nature remains neutral with regard to jurisdictional claims in published maps and institutional affiliations. 